\title{
Enzymatic hydrolysis of granular native and mildly heat-treated tapioca and sweet potato starches at sub-gelatinization temperature.
}

\begin{abstract}
The effect of mild heat treatment (below gelatinization temperature) towards the susceptibility of granular starch to enzymatic hydrolysis was investigated. Tapioca and sweet potato starches were subjected to enzymatic hydrolysis with a mixture of fungal $\alpha$-amylase and glucoamylase at $35{ }^{\circ} \mathrm{C}$ for $24 \mathrm{~h}$. Starches were hydrolyzed in native (granular) state and after heat treatment below gelatinization temperature $\left(60{ }^{\circ} \mathrm{C}\right.$ for $\left.30 \mathrm{~min}\right)$. The dextrose equivalent (DE) value of heat-treated starch increased significantly compared to native starch, i.e., 36-50\% and 27-34\% for tapioca and sweet potato starch, respectively. Scanning electron microscopy examination showed that enzymatic erosion occurred mainly at the surface of starch granules. Hydrolyzed heat-treated starch exhibited rougher surface and porous granules compared to native starch. X-ray analysis suggested that enzymatic erosion preferentially occurred in amorphous areas of the granules. The amylose content, swelling power and solubility showed insignificant increase for both starches. Evidently, heating treatment below gelatinization temperature was effective in enhancing the degree of hydrolysis of granular starch.
\end{abstract}

Keyword: Starch; Granule; Enzyme hydrolysis; $\alpha$-Amylase; Glucoamylase; Reducing sugar. 